\title{
Relationship between acculturation and blood pressure in Somali immigrants living in Minneapolis-St. Paul, Minnesota
}

\author{
Ahmed Dalmar ( $\nabla$ ahmed.dalmar@aurora.org ) \\ Aurora Health Care https://orcid.org/0000-0002-9891-5852
}

Brian C. Martinson

HealthPartners Institute for Education and Research

Morgan Brown

HealthPartners Institute for Education and Research

Maharaj Singh

Aurora Health Care

Douglas Pryce

Hennepin County Medical Center

Michael V. Maciosek

HealthPartners Institute for Education and Research

Farhiya Farah

HealthPartners Institute for Education and Research

Miles Ott

Fife College

Bjorn Westgard

HealthPartners Institute for Education and Research

Research article

Keywords: HTN, Zip codes, immigrant, refugee, acculturation

Posted Date: August 19th, 2019

DOl: https://doi.org/10.21203/rs.2.13115/v1

License: (c) (i) This work is licensed under a Creative Commons Attribution 4.0 International License.

Read Full License 


\section{Abstract}

\section{Background}

Immigrant acculturation to the United States has been found to correlate with cardiovascular risks. Little extant research has evaluated the relationship between acculturation and blood pressure in Somali immigrants.

\section{Methods}

We surveyed and measured blood pressures of 1156 Somali immigrants in Minneapolis-St. Paul, Minnesota. Latent class analysis identified four distinct acculturation subgroups. We examined the data for predictors of hypertension using generalized estimating equations.

Results

Our sample was majority female (62.4\%), mean age $47.9 \pm 18.4$ years, mean baseline body mass index $30.2 \pm 8.6 \mathrm{~kg} / \mathrm{m} 2$ and mean years in U.S. of $9.9 \pm 6.1$. Multivariate regression showed that one acculturation group (low English, high trust) was less likely to be hypertensive.

Conclusion

We found no relationship between several measures of acculturation and hypertension. We found a difference between one of our acculturation groups and the others, suggesting a more complex relationship between acculturation and hypertension among Somali immigrants.

\section{Background}

Acculturation has been implicated in the development of hypertension (HTN) in immigrants from different continents $[1,5]$,but a paucity of studies examines the effect of acculturation on blood pressure (BP) in African immigrants/refugees. African immigrants are one of the fastest-growing segments of the black immigrant population in the U.S, increasing from 881,000 in 2000 to 2.1 million living in the United States in 2015 [6]. Somali immigrants make up almost half of all African refugees arriving in the U.S. Of these, $40 \%$ have settled in the Minneapolis-St. Paul metropolitan area [6, 7].The high influx of these immigrants/refugees, combined with the high prevalence of HTN in the USA, particularly in African Americans, raises public health concerns regarding increased HTN, subsequent cardiovascular disease burdens, and health care costs $[8,9]$.

Although immigrants typically enjoy significant health advantages upon settling in the U.S $[10,15]$, this advantage dissipates quickly as they acquire predominant lifestyle habits, predisposing them to the diseases of affluent societies like HTN $[16,17]$. Often referred to as the "healthy immigrant effect," this process is typically linked to processes of "acculturation." The term acculturation "defines those phenomena which result when groups of individuals of differing cultures come into continuous contact, 
with subsequent changes in the original culture patterns of either or both groups $[18,20]^{\prime \prime}$. Researchers have linked acculturation to health status and behaviors among immigrants [21, 22]. CommodoreMensah et al. [23] in their Afro-Cardiac study provided a foundation for future studies addressing acculturation and CVD risk. Daramola [24] et al. showed some of the associations between migration and cognitive representations of HTN.

\section{Conceptual framework}

We adopted Berry's two-dimensional acculturation model [25] and Schumann's second language acquisition model [26] to inform our conceptual framework as shown in (Fig 1 A and B).

Berry's model describes how people adapt to a new culture in terms of 2 dimensions: 1) How much does the person seek to maintain connection with his or her culture of origin? 2) How much does the person want to learn and adopt the new culture? The two dimensions used to measure acculturation yield a 2by -2 table describing four ways people may adjust to a new culture [25] (Fig 2). We apply this framework to two alternative dimensions: trust and English language proficiency.

Schumann's acculturation model proposes that language is the most potent factor in successfully acculturating. The model suggests that the degree to which second-language learners acculturate themselves towards the culture of a target-language group (TL) generally depends on social and psychological factors; and these two kinds of factors will determine respectively the level of social and psychological "distance." [27, 29] Schumann identifies eight factors that influence social distance and three factors that influence psychological distance [26].(Fig 1 B).

In our study, we therefore sought to measure multiple factors potentially relevant to the acculturation process, including:

- trust as a surrogate for openness, which is operative in adaptation and acculturation,

- English language proficiency as a determinant factor of acculturation,

- duration in U.S. as a crude proxy for length of exposure to the host culture, and

- geographic residence in the zip codes where early refugees/immigrants initially settled in the early 2000s, essentially an enclave, as a proxy for exposure to the culture of origin compared to the host culture.

We acknowledge as a limitation of our study that, while acculturation clearly represents dynamic processes that play out over time, our cross-sectional measures capture and represent just a snapshot in time of these processes. We hypothesized that blood pressure would vary significantly across groups differing in acculturation as identified by English language use and measures of trust.

\section{Methods}


From October 2014 through June 2016, we conducted a cross-sectional study of the prevalence of cardiometabolic disease risk in a sample of 1156 community-dwelling Somalis, age $\geq 18$ years, living in the Minneapolis-St. Paul metropolitan area of Minnesota, using respondent-driven sampling (RDS) [37] to recruit participants. RDS is a chain-referral recruitment process appropriate for use in "hidden" populations or those for which no adequate sampling frame exists. The recruited social contacts of the first wave of respondents become respondents and recruiters of the second wave, and so on. Sampling continues for between 5 and 9 waves, leading to an approximation of a random sample [30, 31]. Trained ethnicity- and gender-concordant community health workers (CHWs) obtained informed consent translated into Somali language for non-English speaking participants, conducted face-to-face interviews, obtained anthropometric and BP measurements. Our study team developed the interview questions in collaboration with an advisory panel of Somali individuals from the local community. The HealthPartners Institutional Review Board approved this study.

\section{Measurements}

We used self-report interview questions to obtain most measures. Anthropometric measures included measured height, weight, waist-circumference and BP. Scales were calibrated before each measurement to reduce reader error. Height was measured by a stadiometer to the nearest $0.5 \mathrm{~cm}$ without footwear. Using finger-stick, blood-spot samples, we obtained (non-fasting) measures of blood-sugar and cholesterol levels.

\section{Primary outcome - hypertension}

For BP measurements, participants rested quietly, seated, for 5 minutes, and once the participant's maximum inflation level was determined, the $\mathrm{CHWs}$ obtained 3 consecutive BP readings by using sphygmomanometer with an appropriately sized arm cuff. The average of 3 systolic blood pressure (SBP) and diastolic blood pressure (DBP) outputs was recorded.

We defined HTN as SBP $\geq 140 \mathrm{~mm} \mathrm{Hg}$ or DBP $\geq 90 \mathrm{~mm} \mathrm{Hg}$, the subject saying "yes" to taking antihypertensive medication, or the subject reporting having been told by a health care provider that they had HTN.

\section{Primary predictors-acculturation}

Our measures of acculturation included:

- Traditional measures (Duration in USA and Enclaved geographically in one of 8 contiguous zip codes (yes, no).

- Proxy measures. Four latent acculturation subgroups: High Trust/Low English, Low Trust/Low English, High Trust/High English, and Low Trust/High English. 
In the context of immigrant communities, "acculturation" references the process of change and adaptation by individuals and groups who have migrated from one location and culture to another. [14, 15] As a process not directly observable, it is useful to consider acculturation as a latent variable, the values of which may be inferred from multiple observed measures. Latent class analysis (LCA) is one appropriate method for identifying latent subgroups. This method identifies exhaustive and mutually exclusive subgroups within a sample, based on evaluation of categorical input measures. We used PROC LCA in SAS 9.4 [38] to identify latent subgroups within our sample based on survey responses.

Our survey included several questions about English language use and proficiency ("Do you speak English, read or write in English, and in what language do you usually read?"), as well as several questions about trust of others ("Can most people be trusted?", "Can you trust Somali people?", and "Can you trust non-Somali people?"). Having coded each of these measures as a "yes or no" response, we conducted LCA using these as inputs, to identify subgroups in our sample, distinguishable based on patterns of response to these questions. Further details of the LCA analyses are beyond the scope of this paper and are being prepared for inclusion in a separate publication.

\section{Geographic Enclave}

We defined individuals as "geographically enclaved" (a 0/1 measure) based on whether, at the time of our current survey, they reported living in one of eight contiguous zip codes in Minneapolis that represented the initial residence locations for most Somali immigrants to Minnesota around the year 2000. We defined individuals living outside of those zip codes as being not enclaved.

\section{Duration in U.S.}

We asked respondents in what year they arrived in the U.S. From this, and their interview date, we computed their duration in the U.S. in years. There were 8 respondents who reporting having been born in the U.S. who we assigned missing values for this measure.

\section{Other Covariates/Confounders}

Other covariates and potential confounder variables included in our multivariate models include continuous age in years, gender (male or female), marital status (not married $=1$, married or in a partnered relationship $=2$, separated/widowed/divorced $=3$ ), poverty threshold (below or above federal poverty level), employment (yes or no), education (more or less than high school education), and smoking status (current smoker, nonsmoker). Social resources as "high" for the group reporting having access to 9 or more of a possible 12 types of social resources or "low" for those reporting having access to 8 or fewer types of support, and physical activity as "active" ( $\geq 150$ minutes/week) and "less active" ( $<150$ minutes/week). Health insurance status was recorded as yes or no, based on self-report. Body mass index was calculated as weight in kilograms divided by height in square meters as a continuous variable. Interview questions included history of diabetes and history of high cholesterol and were categorized as 
yes or no. We defined as diabetic those with hemoglobin $\mathrm{A} 1 \mathrm{C} \geq 6.5$ percent according to National Institute of Diabetes and Digestive and Kidney Diseases. We defined dyslipidemia as a ratio of Cholesterol/ High density lipoprotein (Chol/HDL $\geq 5$ ).

To aid interpretation of our multivariate modeling results, we centered age and duration of residence (years) in the U.S. by computing separate variables that subtract the sample average age and duration in U.S. values, respectively, from everyone's age and duration of residence in the U.S.

\section{Statistical Analyses}

Analysis of RDS data requires sample weights based on an individual's likelihood of sample inclusion, a function of the size of their social network, since individuals with more network contacts have a higher probability of being included in the sample. We used standard weights generated by RDSAT 7.1 software to re-equalize sample inclusion probabilities across individuals in the sample [30,31].

Because individuals who are directly linked within a social network are more likely to share characteristics, RDS data also results in non-independence, or correlated data. To account for the correlated observations inherent in RDS data, as well as the unequal sampling probabilities, we estimated the relationships between HTN and our measures of acculturation using an adaptation of Generalized Estimating Equations (GEE) with a multivariate logistic regression model. We estimated correlations between individuals who were directly connected in the respondent driven sampling referrals, using the weights from RDSAT in the GEE to account for the unequal sampling probabilities [32].

We describe the characteristics of the study population by frequency (percentage) and mean ( \pm standard deviation). We assessed comparisons between groups using Student's t-test and ANOVA for continuous variables and by chi-squared and/or Fisher's exact test wherever appropriate for categorical data. We performed the descriptive analyses using SAS Version 9.4 (SAS Institute Inc., Cary, NC.). In multivariate logistic regressions we considered P-values of $<0.05$ statistically significant. For our multivariate analyses we used the geeNET package (citation to https://github.com/MilesOtt/geeNET) in R [33].

\section{Results}

\section{Study population}

We present descriptive statistics about our sample in (Table 1). A total of 1,156 were included in the study. Most participants were women (63.0\%). The mean age was $47.9 \pm 18.4$ years (median 46.0, range 18-90), mean baseline BMI was $30.0 \pm 8.7 \mathrm{~kg} / \mathrm{m}^{2}$ (median 29.4, range 14.0-92.1), and mean years in U.S. was $9.9 \pm 6.1$ (median 10.0, range $0-44$ ). Overall, $80 \%$ of participants had health insurance, $46.4 \%$ (536/1156) had dyslipidemia, and 21\% (243/1156) had diabetes. Most were below poverty level. Approximately half of the sample were either married or in a partnered relationship, while just under a third were widowed, separated, or divorced. Of our total sample, $70 \%$ lived in the eight contiguous zip codes. 
A total of $29.5 \%$ (341) of participants had HTN, $84 \%$ (287/341) of whom were on medication. Of patients on medication, $38 \%[1.00-(178 / 287=0.62 \%)]$ had controlled HTN, compared to $48.0 \%$ [1-(178/341)] of participants who were aware of their HTN. BP was classified with the following parameters: normal BP = $52.2 \%(603 / 1156)$, pre-hypertension = $31.8(368 / 1156)$, stage $1 \mathrm{HTN}=11.8(136 / 1156)$, and stage $2 \mathrm{HTN}$ $=3.6 \%$ (42/1156). We did not find a correlation between systolic blood pressure and duration of residence in U.S (Fig 2A). But Similar to the general U.S. population, women were equally as likely as men to have HTN. However, for individuals under 45 years old, the condition affects more men than women. For individuals 65 years old or older, high BP affected more women than men (Figure 3)

\section{Predictors of HTN}

Results of our multivariate logistic regression models are presented (Table 2). The effective $\mathrm{N}$ differs from model, with between 242 and 267 observations being deleted due to missing data, depending on model. After controlling for gender, age, geographic enclave residence, duration of residence in U.S.A, insurance, education, smoking, employment, activity levels, marital status, poverty level, BMI, social resources score, dyslipidemia, and diabetes mellitus, we observed that, relative to the acculturation group with Low Trust-Low English, the acculturation group with High Trust-Low English were significantly less likely to have HTN (OR 0.45, C.I 0.24-0.85, p = 0.0115). In contrast, both the High Trust-High English group and the Low Trust-High English acculturation groups had likelihoods of HTN that did not differ significantly from the comparison group.

We also examined other potential indicators of acculturation. Duration of residence and employment status are other potential indicators of acculturation and they showed no association with HTN, regardless of whether our other measures of acculturation were also included in the model. Diabetes was an independent predictor of HTN, $(\mathrm{OR}=1.88, \mathrm{C} . \mathrm{I} 1.14-3.12 \mathrm{p}=0.0036)$.

\section{Discussion}

While the healthy immigrant effect has been demonstrated in multiple populations and conditions, to date the consensus has been less definite about the role of acculturation or lack of it on blood pressure. Many observational studies report strong associations between Western acculturation and HTN. Moran et al. [2] reported a corollary result that being less acculturated to the United States mainstream culture was associated with a decreased prevalence of HTN. In this study, we evaluated the relationship between acculturation and blood pressure in Somali refugees/immigrants living in metropolitan Minneapolis-St. Paul. In our study, acculturation itself does not appear to be strongly associated with increased hypertension among Somali immigrants, but rather protective from it in the High Trust-Low English group, findings that are more consistent with those of Moran et al. [2].

Refugees/immigrants acculturate differently depending on many factors. It might be supposed that Somalis living in the eight contiguous zip codes where early refugees/immigrants initially settled in the 
early 2000s would acculturate less. According to the healthy immigrant effect, we would then expect less HTN in those individuals. However, we did not find any association between residency in the original ethnic enclave and the presence or lack of HTN. We also did not find a relationship between any of the acculturation groups and the presence of HTN. However, one of our acculturation groups, the Low English-High Trust group was associated with the absence of HTN. According to the Schumann model, language is the largest factor in the acculturation process and its success depends on social and psychological factors. We would thus expect low English proficiency to be associated with less acculturation. The majority of Somalis in our study did not speak English upon arrival, and $72.2 \%$ had low English proficiency, which can be considered a potential marker of less acculturation.

If having low English proficiency is a marker of less acculturation, then it is also possible that having high trust in that setting enables one to benefit most from the relatively tight knit social connections and mutual assistance within the Somali community. Because of cultural and religious differences, Somalis in the U.S. do not share churches, clubs, and recreational facilities with the local population, which inhibits contacts and slows the pace of acquisition of English language and acculturation. Somalis are highly interconnected and tend to have less contact with their host group; they also differ extremely in culture, which inhibits acculturation and contact. But preserving their culture through tight knit families and community with high social support may make it easier for them to handle the stresses of acculturation and life in the U.S. environment. The lower prevalence of HTN in Low English-High Trust individuals can thus be construed as indirect evidence of the corollary of the healthy immigrant effect [2].

While participants in this study maintained their collective or group level acculturation, individual psychological acculturation may have resulted in a higher prevalence of overweight/obesity scores (75\% compared to U.S. average of $70 \%$ ) and diabetes $20.1 \%$ (232/1151) compared to U.S. average of $9.4 \%$. The higher prevalence, particularly in women, is likely due to an increase in the availability of high-calorie foods even in their ethnic food groceries and a decrease in physical activity levels (only $20 \%$ reached $\geq 150$ minute/week activity) (Table 1). Similar results were found in studies from many African countries particularly among urban women $[34,35]$. Of note, BMI was not associated with SBP in current and in our 2000 study [36] (Fig 2B).

Limitations: The main limitation of our study is its observational design (cross-sectional), which limits our ability to demonstrate causation. This also means that our measures of the acculturation "process" only capture that process at an arbitrary point in time for each respondent, and do not inform us as to where in the process each respondent may be at the point of interview. We do not have sufficiently detailed information regarding diet in our Somali sample to estimate its contribution to HTN and CVD risk factors. Unfortunately, there is also no available validated acculturation survey scale for Somali speaking communities. To the extent that our RDS-based sample represents the local Somali community, our results should be generalizable to the population of Somalis residing in the Minneapolis-St. Paul area. We do not know whether our results are generalizable beyond this.

\section{Conclusions}


This study contributes to the literature by suggesting that some degree of the healthy immigrant effect is at work in the development of HTN among Somali refugees and immigrants in the U.S. The prevalence of HTN in the cohort from this study is lower than that of the U.S. population and Africans living in urban areas. Low English proficiency and High Trust, characteristics that are likely to be most protective for the stresses of acculturation, are associated with a lower chance of HTN. These results are important for designing and implementing health promotion and education programs to prevent HTN and other cardiometabolic risk factors in immigrant and refugee communities that can take advantage of the protective elements of the culture of origin while facilitating engagement and acculturation with the receiving culture.

\section{Declarations}

\section{Ethics approval and consent to participate}

HealthPartners Institutional Review Board approved this study prior to any subject recruitment or data collection taking place. All subjects provided written informed consent prior to study participation.

\section{Consent for publication}

Not applicable

\section{Availability of data and materials:}

Our study population is an immigrant community in which a large number of individuals are refugees, and against which biases have sometimes been expressed. There is therefore particular sensitivity in the community to the confidentiality of individual responses and data. To comply with the assurances made to the study participants, and to the overseeing IRB that participant data would not be shared beyond the study team, the authors are limited to sharing data as aggregated for the presented analyses. These restrictions notwithstanding, the authors confirm that the appropriately aggregated data will be made available upon appropriate request to interested researchers, pending ethics approval. Those wishing to access study data for such analyses may contact the corresponding author.

\section{Competing interests}

The Authors have no competing interests

\section{Funding}

Research reported in this publication was supported by National Heart, Lung, and Blood Institute of the National Institutes of Health. The content is solely the responsibility of the authors and does not 
necessarily represent the official view of the National Institutes of Health. The funder had no involvement in the design of the study, the collection, analysis and interpretation of the data, nor in writing the manuscript.

\section{Authors' contributions}

Conceived and designed the research: $B W, B M, M M, D P, A D$

Performed statistical analysis: BM, AD, MS, MO

Acquired the data: BM, BW, MM, FF

Drafted the manuscript: AD, BM, BW,

Made critical revision of the manuscript for key intellectual content: BW, AD, BM, MM, DP, FF, MO

\section{Acknowledgement}

The authors are grateful to WellShare International (Minneapolis, MN) and East Africa Health Project (St. Paul. MN) for recruitment, anthropometric measurement, blood testing and data collection; we also thank publication support staff at the Aurora Research Institute (Milwaukee, WI) for editorial preparation of the manuscript.

\section{Abbreviations}

$\mathrm{OR}=$ odds ratio. $\mathrm{BP}=$ blood pressure. Duration in US centered = duration of residence (years) in USA centered. See last paragraph of page 9 . Coeff = coefficient; $\mathrm{OR}=$ odds ratio; $\mathrm{Cl}=$ confidence interval; Accul4 = acculturation category; Diab_A1C, diabetes, Hgb A1C; dyslip = dyslipidemia (Cholesterol/ High density lipoprotein (Chol/HDL $\geq 5$ ). poverty threshold = definition of poverty in USA

\section{References}

1. Dallo FJ, James SA. Acculturation and blood pressure in a community-based sample of ChaldeanAmerican women. J Immigr Health. 2000;2(3):145-153. doi: 10.1023/A:1009560903668 http://www.ncbi.nlm.nih.gov/pubmed/16228747

2. Moran A, Diez Roux AV, Jackson SA, et al. Acculturation is associated with hypertension in a multiethnic sample. Am J Hypertens. 2007;20(4):354-363. doi: 10.1016/j.amjhyper.2006.09.025. http://www.ncbi.nlm.nih.gov/pubmed/17386340

3. Padilla AM. Acculturation. Theory, Model and some New Findings; Westview Press;1980

4. Steffen PR, Smith TB, Larson M, Butler L. Acculturation to Western society as a risk factor for high blood pressure: a meta-analytic review. Psychosom Med. 2006;68(3):386-397. doi 
10.1097/01.psy.0000221255.48190.32 http://www.ncbi.nlm.nih.gov/pubmed/16738069

5. Tailakh AK, Evangelista LS, Morisky DE, Mentes JC, Pike NA, Phillips LR. Acculturation, Medication Adherence, Lifestyle Behaviors, and Blood Pressure Control Among Arab Americans. J Transcult Nurs. 2016;27(1):57-64. doi: 10.1177/1043659614526456. Epub 2014 May 20.

http://www.ncbi.nlm.nih.gov/pubmed/24848347

6. Anderson M. African immigrant population in U.S. steadily climbs. Pew Research Center; 2015. http://pewrsr.ch/2I55fhT

7. Njeru JW, Tan EM, St Sauver J, et al. High Rates of Diabetes Mellitus, Pre-diabetes and Obesity Among Somali Immigrants and Refugees in Minnesota: A Retrospective Chart Review. J Immigr Minor Health. 2016;18(6):1343-1349. doi: 10.1007/s10903-015-0280-3.

http://www.ncbi.nlm.nih.gov/pubmed/26416285

8. Chobanian AV, Bakris GL, Black HR, et al. Seventh report of the Joint National Committee on Prevention, Detection, Evaluation, and Treatment of High Blood Pressure. Hypertension. 2003;42(6):1206-1252. doi: 10.1161/01.HYP.0000107251.49515.c2. Epub 2003 Dec 1. http://www.ncbi.nlm.nih.gov/pubmed/14656957

9. Benjamin EJ, Blaha MJ, Chiuve SE, et al. Heart Disease and Stroke Statistics 2017 Update: A Report From the American Heart Association. Circulation. 2017;135(10):e146-e603. doi:

10.1161/CIR.0000000000000485. Epub 2017 Jan 25.

http://www.ncbi.nlm.nih.gov/pubmed/28122885

10. Cunningham SA, Ruben JD, Narayan KV. Health of foreign-born people in the United States: a review. Health \& place. 2008;14(4):623-635. doi: 10.1016/j.healthplace.2007.12.002. Epub 2008 Jan 31.

11. Egolf B, Lasker J, Wolf S, Potvin L.The Roseto effect: a 50-year comparison of mortality rates. Am J Public Health. 1992;82(8):1089-1092. http://www.ncbi.nlm.nih.gov/pubmed/1636828 doi: 10.2105/ajph.82.8.1089.

12. Kennedy S, Kidd MP, McDonald JT, Biddle N. The healthy immigrant effect: patterns and evidence from four countries. Journal of International Migration and Integration. 2015;16(2):317-332. https://doi.org/10.1007/s12134-014-0340-x

13. Ng E. The healthy immigrant effect and mortality rates. Health Rep. 2011;22(4):25-29. http://www.ncbi.nlm.nih.gov/pubmed/22352149

14. Venters H, Gany F. African Immigrant Health. J Immigr Minor Health. 2011;13(2):333-344. doi: 10.1007/s10903-009-9243-x. Epub 2009 Apr 4. http://www.ncbi.nlm.nih.gov/pubmed/19347581

15. Walker P, Barnett, E. An introduction to the refugee and immigrant healthcare. Immigrant Medicine 1st Edition - ISBN: 9780323034548, 9780323070577. Imprint: Saunders 2007. EBOOK.

16. Antecol H, Bedard K. Unhealthy assimilation: why do immigrants converge to American health status levels? Demography. 2006;43(2):337-360. doi: 10.1353/dem.2006.0011. http://www.ncbi.nlm.nih.gov/pubmed/16889132

17. Kandula NR, Kersey M, Lurie N. Assuring the health of immigrants: what the leading health indicators tell us. Annu Rev Public Health. 2004;25:357-376. doi: 
10.1146/annurev.publhealth.25.101802.123107. http://www.ncbi.nlm.nih.gov/pubmed/15015925

18. Redfield R. Memorandum on the study of acculturation. Am Anthropol. 1936;38:149-152. American anthropologist, 1936 - Wiley Online Library

19. Berry JW. Acculturation and adaptation. health consequences of culture contact among circumpolar peoples. Arctic Med Res. 1990;3:142-150. http://www.ncbi.nlm.nih.gov/pubmed/2206174

20. Berry JW. Acculturation: Living successfully in two cultures. International Journal of Intercultural Relations. 2005;29:697-712. https://doi.org/10.1016/S0147-1767(99)00009-7

21. Berry JW. Contexts of acculturation. New York: Cambridge University Press. 2006 Contribution of a Book.

22. Castro V. Acculturation and psychological adaptation. Westport, CT, US: Praeger Publishers/Greenwood Publishing Group.2003.

23. Graves T. Psychological acculturation in a tri-ethnic community. South-Western Journal of Anthropology winter. 1967;23 (4):337-350). https://doi.org/10.1086/soutjanth.23.4.3629450

24. Berry JW. Marginality, stress and ethnic identification in an acculturated Aboriginal community. Journal of Cross-Cultural Psycholog. 1970;1(239-252).

https://doi.org/10.1177/135910457000100303

25. Lara M, Gamboa C, Kahramanian MI, Morales LS, Bautista DE. Acculturation and Latino health in the United States: a review of the literature and its sociopolitical context. Annu Rev Public Health. 2005;26:367-397. doi: 10.1146/annurev.publhealth.26.021304.144615.

http://www.ncbi.nlm.nih.gov/pubmed/15760294

26. Salant T, Lauderdale DS. Measuring culture: a critical review of acculturation and health in Asian immigrant populations. Soc Sci Med. 2003;57(1):71-90.

http://www.ncbi.nlm.nih.gov/pubmed/12753817

27. Commodore-Mensah Y, Sampah M, Berko C, et al. The Afro-Cardiac Study: Cardiovascular Disease Risk and Acculturation in West African Immigrants in the United States. Rationale and Study Design. J Immigr Minor Health. 2016;18(6):1301-1308. doi: 10.1007/s10903-015-0291-0. http://www.ncbi.nlm.nih.gov/pubmed/26429573

28. Daramola OI, Scisney-Matlock M. Migration and cognitive representations of hypertension in African immigrant women. West J Nurs Res. 2014;36(2):209-227. doi: 10.1177/0193945913504501. Epub 2013 Sep 11. http://www.ncbi.nlm.nih.gov/pubmed/24025222

29. Berry JW. Immigration, Acculturation, and Adaptation. Applied Psychology1997; 46(1):5-34. https://onlinelibrary.wiley.com/doi/abs/10.1111/j.1464-0597.1997.tb01087.x

30. Schumann J. Research on the acculturation model for second language acquisition. Journal of Multilingual and Multicultural Development. 1986;7(5):379-392.

https://doi.org/10.1080/01434632.1986.9994254

31. Schumann J. The Pidginization Process: A Model for Second Language Acquisition. Rowley, MA. Newbury House 1978. https://doi.org/10.1017/S0272263100004216 
32. Brown HD. TESOL Quarterly. 1980;14(2):157-164. DOI: 10.2307/3586310

https://www.jstor.org/stable/3586310

33. Ellis R.The Study of Second Language Acquisition. Oxford University; 1994.

34. Heckathorn DD. Respondent-Driven Sampling: A New Approach to the Study of Hidden Populations, Social Problems. 1997;44(2):174-199 https://doi.org/10.2307/3096941

35. Salganik MJ, Heckathorn DD. Sampling and estimation in hidden populations using respondentdriven sampling. Sociological Methodology. 2004;34:193-239) https://doi.org/10.1111/j.00811750.2004.00152.x

36. Volz E, Heckathorn DD. Probability Based Estimation Theory for Respondent-Driven Sampling. Journal of Official Statistic. 2008;24 (1):79-97

37. Ott M: https://github.com/MilesOtt/geeNET) in R.

38. Vijver Svd. Status report on hypertension in Africa - Consultative review for the 6th Session of the African Union Conference of Ministers of Health on NCD. The Pan African Medical Journal. 2013;16.

39. Opie LH, Seedat YK. Hypertension in sub-Saharan African populations. Circulation. 2005;112(23):3562-3568. https://doi.org/10.1161/CIRCULATIONAHA.105.539569

40. Dalmar A. OVERWEIGHT AND OBESITY IN SOMALI IMMIGRANTS TO USA. Ethn Dis. 2006;16:S4-S35.

\section{Tables}

Due to technical limitations, Tables $1 \& 2$ are only available for download from the Supplementary Files section.

\section{Figures}

Fig 1A. Berry's Acculturation Model

Cultural Adaptation

(relationship sought among groups)

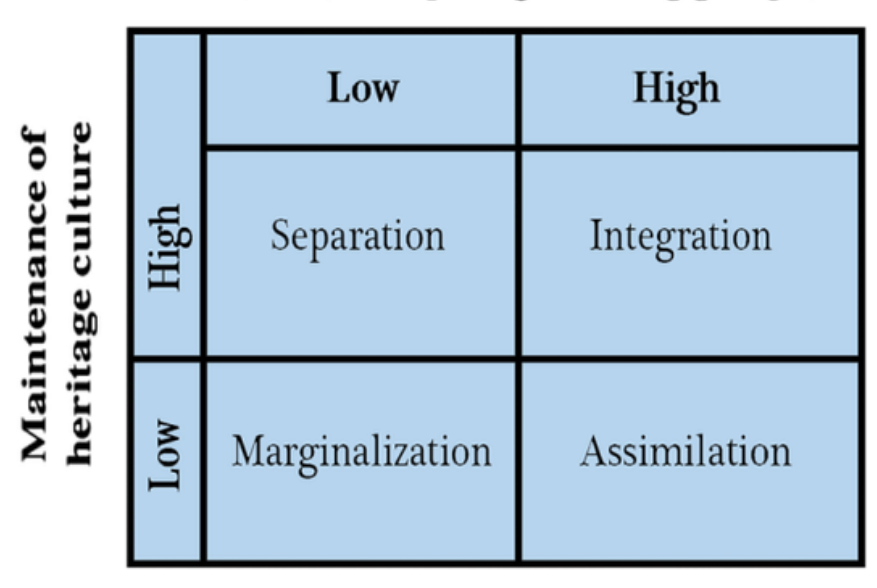

Fig 1B. Schumann's Acculturation Model
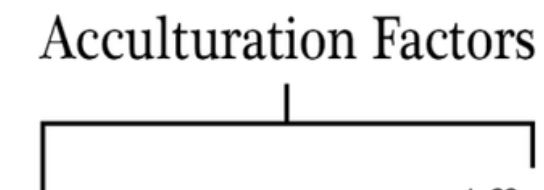

Social

Factors

Dominance, Non-dominance, subordination, assimilation, acculturation, preservation, cohesiveness, attitude, intended length of the residence of TL area.
Affective Factors (psychological variables )

Language shock, culture shock, motivation egopermeability 
Figure 1

A. Berry's Acculturation Model; B. Schumann's Acculturation Model

Fig 2. Bivariate scatter plot of systolic blood pressure by duration in USA and by Mass Body Index
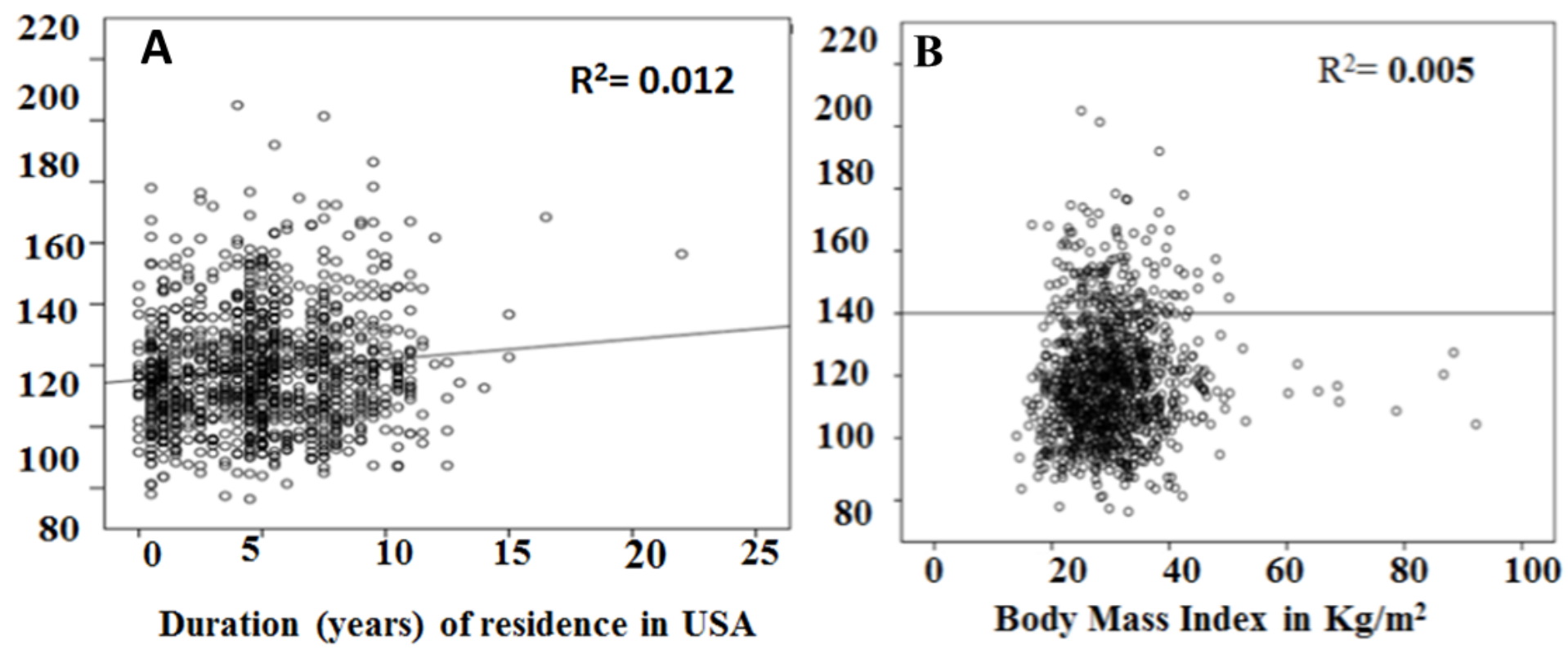

Figure 2

Bivariate scatter plot of systolic blood pressure by duration in USA and by Mass Body Index 


\section{Fig 3. Prevalence of HTN by age and gender}

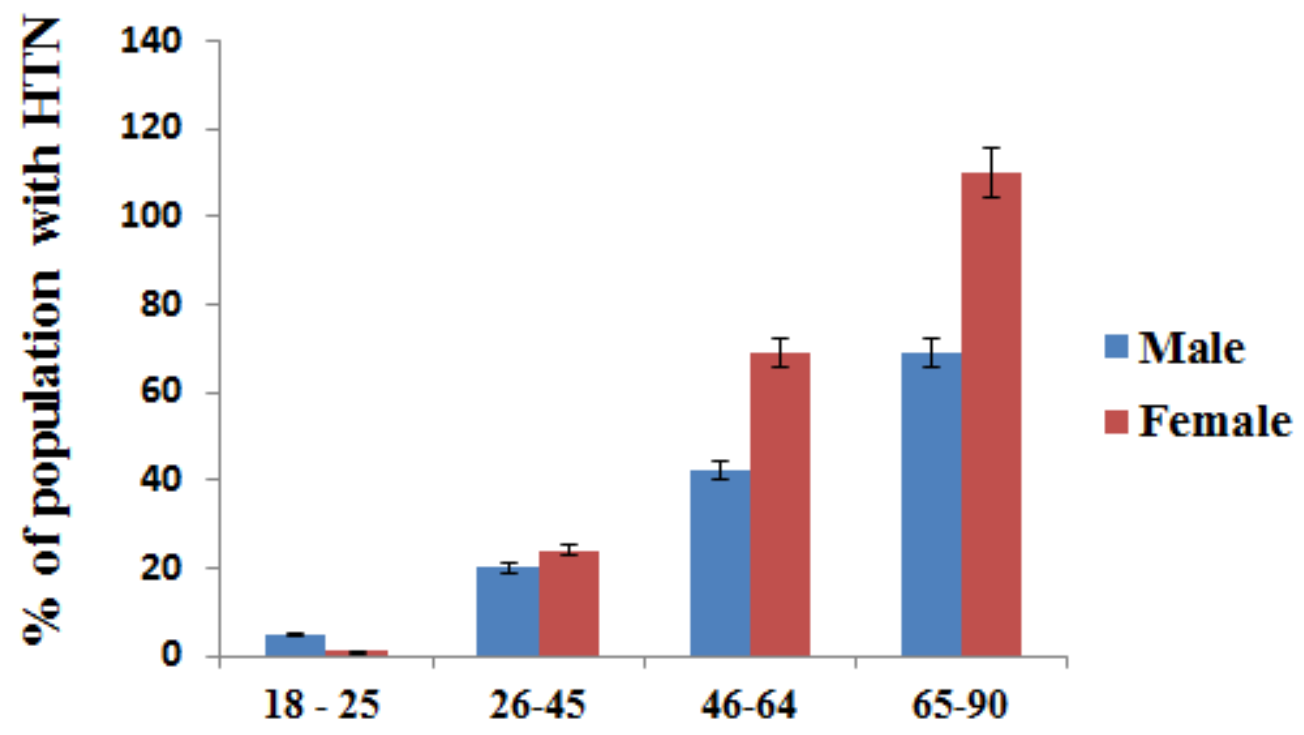

$\begin{array}{llllr}\text { Male } & 5 & 20 & 42 & 69 \\ \text { Female } & 1 & 24 & 69 & 110\end{array}$

Figure 3

Prevalence of HTN by age and gender

\section{Supplementary Files}

This is a list of supplementary files associated with this preprint. Click to download.

- Table2.pdf

- STROBEStatement.pdf

- Table1.pdf 